\title{
New Ways of Thinking about Proteinuria and Progression of Renal Damage
}

\author{
Y.S. Zhou A.N. Turner \\ MRC Centre for Inflammation, University of Edinburgh, Edinburgh, UK
}

Reducing proteinuria is often a target of treatment because of the close correlation between improvement of proteinuria and better renal outcomes in human studies involving angiotensin-converting enzyme inhibitors (ACEI) and angiotensin receptor blockers (ARB).

Conventional explanations for the protective effects of ACEI and ARB centre on their haemodynamic effects, suggesting that reducing glomerular filtration pressure is the key to their protective action. The fact that blood pressure reduction by any means protects renal function in proteinuric renal disease (the Modification of Diet in Renal Disease Study) suggests that this can explain at least some of the effect. Others have added that ACEI/ ARB may also modulate the toxicity of filtered proteins by effects on non-glomerular cells [1]. The close correlation between long-term outcomes and proteinuria has triggered a large body of work documenting potential mechanisms by which excessive filtered protein may damage renal tubular cells and promote interstitial fibrosis [2].

An alternative hypothesis is emerging from the mushrooming field of podocyte biology. Podocytes have been show to play a central role in many or most proteinuric diseases - almost all of the inherited causes of severe proteinuria involve podocyte genes and many acquired proteinuric diseases can point to podocyte malfunction as well. Proteinuria is known to be a consequence of podocyte malfunction or damage.

\section{KARGER}

Fax +4161306 1234 E-Mail karger@karger.ch www.karger.com
(C) 2010 S. Karger AG, Basel

$1660-2129 / 10 / 1161-0001 \$ 26.00 / 0$

Accessible online at:

www.karger.com/nee
The 'Podocyte-Progression hypothesis' [3-5] postulates that continuing, progressive podocyte damage or loss is central to the progression of proteinuric renal diseases. This mechanism of progression might also be extended to diseases in which podocyte damage is not the primary problem, but in which proteinuria occurs later, possibly in hyperfiltering glomeruli, for example.

If we believe this, we might expect nephroprotective, proteinuria-lowering treatments to act not via haemodynamic or anti-fibrotic effects, but on podocytes themselves. Evidence to support this hypothesis is now strong. It turns out that podocytes are dynamic, active, mobile cells that are studded with scores of receptor types enabling them to respond to external mediators. The slit diaphragm is not a static barrier to protein; it is susceptible to a wide range of external influences both chemical and mechanical.

Five years ago came the first suggestion that one of our key immunosuppressive agents, corticosteroids - the mainstay of therapy for minimal change nephrotic syndrome - might have direct structure-protective effects on podocytes [6]. That has recently been added to by the observation that the immunosuppressive calcineurin inhibitor tacrolimus has similar direct effects $[7,8]$. Both of these drugs had previously been assumed to be acting through the immune system, though that fitted poorly with the observation that calcineurin inhibitors seemed to reduce proteinuria regardless of the cause. The evi- 
dence that minimal change nephropathy is an immunemediated condition now looks seriously weakened. It is fascinating that drugs acting on the immune system can have such effects on podocytes and this must point to shared signalling pathways.

It is logical to ask next whether other drugs that appear to reduce proteinuria, or maintain remissions in nephrotic syndrome, could have direct actions on podocytes. In this issue, Takeuchi et al. [9] set out to investigate the imidazole nucleoside mizoribine, which is an inhibitor of purine nucleotide synthesis that is apparently commonly used as an immunosuppressant in Japan.

The chosen model was puromycin aminonucleoside (PAN) nephropathy in rats. PAN is believed to act primarily as a podocyte toxin in this model, causing proteinuria and podocyte injury. Mizoribine acted to protect podocytes in vitro against loss of actin structure and cell death induced by puromycin and in the in vivo model, it reduced proteinuria and preserved nephrin distribution pattern at 9 days. Unfortunately, this is the limit of the in vivo data but a protective effect has been described previously by another group. Some observations on potential mechanisms are presented and it is interesting that the investigators found that there was no evidence in their in vitro system to support a mechanism involving inhibition of inosine $5^{\prime}$-monophosphate dehydrogenase, thought to be the main immunosuppressive action of the drug.

Interestingly or surprisingly, in this same in vitro system the investigators found no protection from mycophenolate mofetil, which is quite extensively used to maintain remission in minimal change disease.

It is only fair to point out that the published randomized controlled trial evidence for the efficacy of mizoribine in nephrotic syndrome is rather limited, and a Cochrane review [10] regarded it as inconclusive. However, the evidence for mycophenolate mofetil is not solid either. Further studies of potential actions of nephroprotective drugs on podocytes are to be looked forward to.

\section{References}

1 Rosenberg ME, Smith LJ, Correa-Rotter R, Hostetter TH: The paradox of the renin-angiotensin system in chronic renal disease. Kidney Int 1994;45:403-410.

-2 Baines RJ, Brunskill NJ: The molecular interactions between filtered proteins and proximal tubular cells in proteinuria. Nephron Exp Nephrol 2008;110:e67-e71.

-3 Gassler N, Elger M, Kränzlin B, Kriz W, et al: Podocyte injury underlies the progression of focal segmental glomerulosclerosis in the Fa/Fa Zucker rat. Kidney Int 2001;60:106116.

4 Ichikawa I, Ma J, Motojima M, Matsusaka T: Podocyte damage damages podocytes: autonomous vicious cycle that drives local spread of glomerular sclerosis. Curr Opin Nephrol Hypertens 2005;14:205-210.
5 Wharram BL, Goyal M, Wiggins JE, et al: Podocyte depletion causes glomerulosclerosis: diphtheria toxin-induced podocyte depletion in rats expressing human diphtheria toxin receptor transgene. J Am Soc Nephrol 2005;16:2941-2952.

6 Ransom RF, Lam NG, Hallett MA, Atkinson SJ, Smoyer WE: Glucocorticoids protect and enhance recovery of cultured murine podocytes via actin filament stabilization. Kidney Int 2005;68:2473-2483.

7 Faul C, Donnelly M, Merscher-Gomez S, Chang YH, Franz S, Delfgaauw J, Chang JM, Choi HY, Campbell KN, Kim K, Reiser J, Mundel P: The actin cytoskeleton of kidney podocytes is a direct target of the antiproteinuric effect of cyclosporine A. Nat Med 2008;14:931-938.
18 Mathieson PW: Proteinuria and immunity an overstated relationship? N Engl J Med 2008;359:2492-2494.

-9 Takeuchi S, Hiromura K, Tomioka M, et al: The immunosuppressive drug mizoribine directly prevents podocyte injury in puromycin aminonucleoside nephrosis. Nephron Exp Nephrol 2010;116:e3-e10.

10 Hodson EM, Willis N, Craig JC: Non-corticosteroid treatment for nephrotic syndrome in children. Cochrane Database of Systematic Reviews: Reviews 2008, Issue 1. DOI: 10.1002/14651858.CD002290.pub3. 